\title{
Verzeichnis der Bearbeiter der 5. Auflage
}

Dr. Johannes Adolff, LL.M. (Cambridge), Rechtsanwalt in Frankfurt am Main, Außerplanmäßiger Professor an der Johann Wolfgang Goethe-Universität Frankfurt am Main

Dr. Michael Arnold, Rechtsanwalt in Stuttgart, Honorarprofessor an der Eberhard Karls Universität Tübingen

Dr. Gregor Bachmann, LL.M. (Univ. of Michigan), Universitätsprofessor an der Humboldt-Universität zu Berlin

Dr. Alfred Bergmann, Vors. Richter am Bundesgerichtshof i.R., Karlsruhe, Honorarprofessor an der Johannes Gutenberg-Universität Mainz

Dr. Tilman Bezzenberger, M.A., Universitätsprofessor an der Universität Potsdam

Dr. Christian Brand, Wissenschaftlicher Assistent Universität Konstanz

Volker Butzke, Rechtsanwalt in Frankfurt am Main

Dr. Christian E. Decher, Rechtsanwalt in Frankfurt am Main, Honorarprofessor an der Johann Wolfgang Goethe-Universität Frankfurt am Main

Dr. Ulrich Ehricke, LL.M. (London), M.A., Richter am Oberlandesgericht a.D., Universitätsprofessor an der Universität zu Köln

Dr. Dr. h.c. Holger Fleischer, Dipl.-Kfm., LL.M. (Univ. of Michigan), Universitätsprofessor, Direktor des Max-Planck-Instituts für ausländisches und internationales Privatrecht, Hamburg

Dr. Max Foerster, LL.M.eur., Privatdozent, Akademischer Oberrat a.Z., Ludwig-Maximilians-Universität München

Dr. Markus Gehrlein, Richter am Bundesgerichtshof a.D.

Dr. Henrik Gildehaus, Rechtsanwalt in Frankfurt am Main

Dr. Dr. Stefan Grundmann, LL.M. (Berkeley), Universitätsprofessor an der Humboldt-Universität zu Berlin

Dr. Mathias Habersack, Universitätsprofessor an der Ludwig-Maximilians-Universität München

Dr. Kai Hasselbach, Rechtsanwalt in München

Dr. Hartwig Henze, Richter am Bundesgerichtshof a.D., Honorarprofessor an der Universität Konstanz

Dr. Heribert Hirte, LL.M. (Berkeley), Universitätsprofessor an der Universität Hamburg, MdB

Dr. Dr. Dr. h.c. mult. Klaus J. Hopt, em. Universitätsprofessor, ehem. Direktor des Max-Planck-Instituts für ausländisches und internationales Privatrecht, Hamburg, vormals Richter am Oberlandesgericht Stuttgart

Dr. Peter M. Huber, Bundesverfassungsrichter, Universitätsprofessor an der Ludwig-MaximiliansUniversität München

Bernadette Kell, Regierungsrätin im Bundesministerium der Justiz und für Verbraucherschutz

Dr. Michael Kort, Universitätsprofessor an der Universität Augsburg

Dr. Katja Langenbucher, Universitätsprofessorin an der Johann Wolfgang Goethe-Universität Frankfurt am Main

Dr. Patrick C. Leyens, LL.M. (London), Professor an der Erasmus Universiteit Rotterdam/Lehrstuhlvertreter an der Universität Hamburg

Dr. Jan Lieder, LL.M. (Harvard), Universitätsprofessor an der Albert-Ludwigs-Universität Freiburg i.Br.

Dr. Hanno Merkt, LL.M. (Univ. of Chicago), Universitätsprofessor an der Albert-Ludwigs-Universität Freiburg i.Br., Richter am Oberlandesgericht Karlsruhe

Dr. Sebastian Mock, LL.M. (NYU), Universitätsprofessor, Wirtschaftsuniversität Wien

Dr. Florian Möslein, Dipl.-Kfm., LL.M. (London), Universitätsprofessor an der Philipps-Universität Marburg

Dr. Peter O. Mülbert, Universitätsprofessor an der Johannes Gutenberg-Universität Mainz

Richard L. Notz, LL.M. (Univ. of Chicago), LL.M. I.B.L. (UCP Lisboa), Rechtsanwalt in Stuttgart

Dr. Hartmut Oetker, Universitätsprofessor an der Christian-Albrechts-Universität zu Kiel, Richter am Oberlandesgericht Jena

Dr. Hans-Joachim Priester, Notar a.D., Honorarprofessor an der Universität Hamburg

Dr. h.c. Volker Röhricht, Vors. Richter am Bundesgerichtshof i.R., Karlsruhe

Dr. Markus Roth, Universitätsprofessor an der Philipps-Universität Marburg

Dr. Alexander Schall, M.Jur. (Oxford), Universitätsprofessor an der Leuphana Universität Lüneburg

Dr. Dr. h.c. mult. Karsten Schmidt, em. Universitätsprofessor an der Rheinischen Friedrich-WilhelmsUniversität Bonn und Professor an der Bucerius Law School Hamburg 
Verzeichnis der Bearbeiter

Dr. Klaus Ulrich Schmolke, LL.M. (NYU), Universitätsprofessor an der Friedrich-Alexander-Universität Erlangen-Nürnberg

Dr. Rolf Sethe, LL.M. (London), Universitätsprofessor an der Universität Zürich

Dr. Felix Steffek, LL.M. (Cambridge), Associate Professor, University of Cambridge

Dr. Dirk Verse, M.jur. (Oxford), Universitätsprofessor an der Ruprecht-Karls-Universität Heidelberg

Dr. Eberhard Vetter, Rechtsanwalt in Köln

Dr. Christine Windbichler, LL.M. (Berkeley), Universitätsprofessorin a.D. an der Humboldt-Universität zu Berlin 